\title{
Video Article \\ Metabolic Labeling and Profiling of Transfer RNAs Using Macroarrays
}

\author{
Sophia Emetu* ${ }^{1}$, Morgan Troiano* ${ }^{*}$, Jacob Goldmintz ${ }^{* 1}$, Jensen Tomberlin ${ }^{1}$, Simon Grelet ${ }^{2}$, Philip H. Howe ${ }^{2}$, Christopher Korey ${ }^{3}$, Renaud Geslain ${ }^{1}$ \\ ${ }^{1}$ Laboratory of tRNA Biology, Department of Biology, College of Charleston \\ ${ }^{2}$ Department of Biochemistry and Molecular Biology, MUSC \\ ${ }^{3}$ Department of Biology, College of Charleston \\ *These authors contributed equally
}

Correspondence to: Renaud Geslain at geslainr@cofc.edu

URL: https://www.jove.com/video/56898

DOI: doi:10.3791/56898

Keywords: Genetics, Issue 131, transfer ribonucleic acids, radioactive orthophosphate, macroarrays, metabolic labeling, gene expression, genetic translation, protein synthesis, non-coding ribonucleic acids

Date Published: 1/16/2018

Citation: Emetu, S., Troiano, M., Goldmintz, J., Tomberlin, J., Grelet, S., Howe, P.H., Korey, C., Geslain, R. Metabolic Labeling and Profiling of Transfer RNAs Using Macroarrays. J. Vis. Exp. (131), e56898, doi:10.3791/56898 (2018).

\section{Abstract}

Transfer RNAs (tRNA) are abundant short non-coding RNA species that are typically 76 to 90 nucleotides in length. tRNAs are directly responsible for protein synthesis by translating codons in mRNA into amino acid sequences. tRNAs were long considered as house-keeping molecules that lacked regulatory functions. However, a growing body of evidence indicates that cellular tRNA levels fluctuate in correspondence to varying conditions such as cell type, environment, and stress. The fluctuation of tRNA expression directly influences gene translation, favoring or repressing the expression of particular proteins. Ultimately comprehending the dynamic of protein synthesis requires the development of methods able to deliver high-quality tRNA profiles. The method that we present here is named SPOt, which stands for Streamlined Platform for Observing tRNA. SPOt consists of three steps starting with metabolic labeling of cell cultures with radioactive orthophosphate, followed by guanidinium thiocyanate-phenol-chloroform extraction of radioactive total RNAs and finally hybridization on in-house printed macroarrays. tRNA levels are estimated by quantifying the radioactivity intensities at each probe spot. In the protocol presented here we profile tRNAs in Mycobacterium smegmatis $\mathrm{mc}^{2} 155$, a nonpathogenic bacterium often used as a model organism to study tuberculosis.

\section{Video Link}

The video component of this article can be found at https://www.jove.com/video/56898/

\section{Introduction}

Cellular tRNA levels fluctuate according to conditions such as cell type, environment, and stress ${ }^{1,2,3}$. SPOt, Streamlined Platform for Observing tRNA, is an original, reliable, and straightforward technique that allows fast and precise quantification of transfer RNA levels in laboratory-grown organisms.

tRNAs have remarkably stable secondary and tertiary structures ${ }^{4}$. They also display numerous post-transcriptional modifications ${ }^{5}$. These features represent significant structural and sequence roadblocks biasing or sometimes preventing direct and reproducible tRNA profiling using standard benchmark RNA quantification techniques ${ }^{6}$. Research groups around the world were forced to develop creative but often convoluted techniques to assess cellular tRNA levels. Some of these methods include: (1) two-dimensional electrophoretic separation of metabolically labeled tRNAs combined with methodical spot assignment by Northern blot ${ }^{1}$; (2) individual quantification by Northern blot using carefully standardized radioactive DNA probes ${ }^{7}$; (3) post-extraction labeling with cyanine fluorochromes followed by microarray analysis ${ }^{3}$, and (4) in vitro stripping of tRNA modifications using recombinant demodification enzymes combined with reverse transcription and subsequent high-throughput sequencing 8 .

SPOt is a simple and original combination of two standard and straightforward approaches: (1) RNA body labeling, which consists in the synthesis, in growing organisms, of radioactive total RNAs and (2) tRNA macroarrays, a systematic and miniaturized genomic tool optimized for tRNA segregation.

Samples are streamlined from living organisms to the quantification platform. They are directly analyzed after extraction and not processed any further which significantly limits biases compared to techniques requiring post-extraction amplification or enzymatic treatments. SPOt is versatile and can be easily combined to polysome fractionation to identify and quantify tRNA subpopulations that are physically associated with translating ribosomes.

The protocol presented here is optimized for Mycobacterium smegmatis, and it was also successfully used to profile tRNAs in Escherichia coli ${ }^{9}$, Saccharomyces cerevisiae ${ }^{10}$, mouse, and human cell cultures ${ }^{11}$. 


\section{Culture and metabolic labeling}

1. Poke once the center of the cap of a sterile $1.5 \mathrm{~mL}$ micro-centrifuge tubes with a 18-gauge needle to allow proper aeration. Inoculate $500 \mu \mathrm{L}$ of supplemented sterile $7 \mathrm{H} 9$ broth with $5 \mu \mathrm{L}$ of $\mathrm{M}$. smegmatis from a starter culture grown overnight ${ }^{12}$.

NOTE: The recipe for one liter of $7 \mathrm{H} 9$ broth is: $4.7 \mathrm{~g}$ Commercial $7 \mathrm{H} 9$ powder, $2 \mathrm{~mL}$ Glycerol, $1 \mathrm{~mL}$ Tween $80,0.85 \mathrm{~g} \mathrm{NaCl}, 5 \mathrm{~g} \mathrm{Albumin}, 2 \mathrm{~g}$ Dextrose, $\mathrm{H}_{2} \mathrm{O}$ (to bring total volume to $1 \mathrm{~L}$ ).

1. (CAUTION) Following standard radioprotection practices, spike the culture media with $20 \mu \mathrm{Ci} / \mathrm{mL}$ of $\left[{ }^{32} \mathrm{P}\right] \mathrm{Na}_{2} \mathrm{HPO}_{4}$. Grow bacteria at $37^{\circ} \mathrm{C}$ and 1,200 rpm agitation, in a shaker incubator placed behind a 9-mm thick acrylic shield.

2. At midlog phase (O.D.600 $\mathrm{nm} \sim 0.5)$, transfer the entire culture into a 2-mL screw-cap tube and pellet radioactive bacteria at room temperature by centrifuging for $2 \mathrm{~min}$ at $10,000 \mathrm{x}$. Collect supernatant containing unincorporated radioactive orthophosphate in appropriate waste container.

\section{Preparation of total RNAs}

NOTE: Total RNAs are extracted from bacterial pellets using a commercial RNA extraction reagent (containing guanidinium thiocyanate, phenol and chloroform) following the manufacturer's protocol.

1. Add $1 \mathrm{~mL}$ of commercial RNA extraction reagent and approximately $200 \mu \mathrm{L}$ of glass beads onto the pellet. Cap the tube securely and disrupt the bacterial in a homogenizer for 2 min under maximum agitation, (setting number five on chosen instrument (see the Table of Materials)).

2. Centrifuge sample at $12,000 \times \mathrm{g}$ for $10 \mathrm{~min}$ at $4{ }^{\circ} \mathrm{C}$. Transfer the supernatant to a new $2-\mathrm{mL}$ tube and discard the beads appropriately. Add $0.2 \mathrm{~mL}$ of chloroform and shake tube vigorously by hand for $15 \mathrm{~s}$. Centrifuge at $12,000 \mathrm{xg}$ for $15 \mathrm{~min}$ at $4{ }^{\circ} \mathrm{C}$.

3. Collect the aqueous phase of the sample into a new tube by angling the tube at $45^{\circ}$. Avoid drawing any of the interphase or organic layer. Add $2 \mu \mathrm{L}$ of colored precipitant and $0.5 \mathrm{~mL}$ of $100 \%$ isopropanol. Shake the tube vigorously by hand for $5 \mathrm{~s}$. Centrifuge at $12,000 \times \mathrm{g}$ for 10 $\min$ at $4^{\circ} \mathrm{C}$.

4. Remove the supernatant from the tube and discard appropriately as the liquid fraction may contain traces of radioactivity. Air dry pellet for 5 min then resuspend in $200 \mu \mathrm{L}$ of $2 \mathrm{X}$ saline-sodium citrate (SSC), $0.1 \%(\mathrm{w} / \mathrm{v}$ ) sodium dodecyl sulfate (SDS) final. NOTE: The composition of stock $20 \mathrm{XSC}$ is $3.0 \mathrm{M} \mathrm{NaCl}, 0.3 \mathrm{M}$ sodium citrate, $\mathrm{pH}$ 7.0.

\section{Preparation of 96 well printer plates}

NOTE: tRNA microarrays are manually printed with forty two 70-mer DNA oligonucleotides complementary to the $3^{\prime}$ end of each M. smegmatis tRNA (terminal CCA excluded) using an eight-pin arrayer. The sequences of the DNA oligonucleotide probes as well as the probe layout in the well-plate and the array are provided (Supplementary File 1- sheet 1 to 3 respectively).

1. Design DNA probes by first retrieving tRNA sequences or tRNA encoding genes from tRNA databases such as the Genomic tRNA Database $^{13}$. Trim conserved $3^{\prime}$ end CCA if encoded. Reverse complement the resulting sequence. Order probe based on the first 70 nucleotides.

2. Resuspend dry DNA probes in water. Adjust probes concentration to $100 \mu \mathrm{M}$. In a 96 well-plate, prepare $120 \mu \mathrm{L}$ of $50 \mu \mathrm{M}$ oligos in $3 \mathrm{X}$ SSC, $0.01 \%(\mathrm{w} / \mathrm{v})$ SDS final (dilute $60 \mu \mathrm{L}$ of stock probes in individual well with $60 \mu \mathrm{L}$ of $6 \mathrm{XSSC}, 0.01 \%(\mathrm{w} / \mathrm{v}) \mathrm{SDS}$.

3. Cover plate with tin foil to prevent evaporation or contamination, freeze immediately and keep at $-20^{\circ} \mathrm{C}$ until needed.

\section{Array printing}

1. Thaw the 96-well plate at room temperature (takes approximately $20 \mathrm{~min}$ ).

2. Label amine-coated glass slides with a diamond pen. Place slides into indexing unit. Follow the grid placements and do as follows.

1. Carefully dip the replicator pins in the wells.

2. Gently print the array on the glass slides, using minimal pressure (you will feel a small resistance). Continue printing without cleaning the arrayer until finished with block $A$.

3. When ready to move on to the next block, follow the washing procedure described below.

1. Dip replicator in the $5 \%$ bleach and gently shake.

2. Press replicator into absorbent paper.

3. Dip replicator in the distilled water, gently shake, and press into paper. Repeat this step once.

4. Dip replicator in isopropanol, gently shake, and press into the paper.

5. Dry the replicator on the fan for about $20 \mathrm{~s}$ before moving on to the next block of the 96 -well plate. Continue to desired number of prints.

4. Allow the slides to dry then crosslink the oligos to the slide using a 254-nm UV crosslinker.

1. Set the energy level to $999,990 \mu \mathrm{J} / \mathrm{cm}^{2}$.

2. Put the slides face up on a clean surface in the crosslinker. Press "start".

5. Block the arrays overnight in $450 \mathrm{~mL}$ of blocking solution on a magnetic stirrer, at room temperature and under slow agitation. Collect and reuse blocking solution up to four times. 
NOTE: The recipe for the blocking solution is: $125 \mathrm{mM}$ phosphate buffer $\left(\mathrm{NaH}_{2} \mathrm{PO}_{4} / \mathrm{Na}_{2} \mathrm{HPO}_{4^{-}}\right) \mathrm{pH} 7.2,2.25 \%$ SDS (w/v), $0.5 \mathrm{mM}$ EDTA, $0.5 \mathrm{X}$ SSC, $1 \%$ BSA (w/v).

6. Wash the slides 2 times for $5 \mathrm{~min}$ at room temperature in $500 \mathrm{~mL}$ of distilled water. Dry the slides by centrifuging for $10 \mathrm{~s}$ at room temperature in a microarray centrifuge. Store arrays in a dry and dark place for up to 6 months.

\section{Array hybridization}

1. Prior to hybridization rinse slides in boiling water and dry by centrifugation (as in 4.6).

2. Place array inside a hybridization cassette and load radiolabeled RNA sample.

3. Run the automated hybridization-washing station for maximum reproducibility.

1. Program steps as following. Condition gaskets for $2 \mathrm{~min}$ at $75^{\circ} \mathrm{C}$. Introduce probe at $60^{\circ} \mathrm{C}$

Denature probes and samples $5 \mathrm{~min}$ at $90^{\circ} \mathrm{C}$. Hybridize samples for $3 \mathrm{~h}$ at $60^{\circ} \mathrm{C}$.

1. Wash slides at $50^{\circ} \mathrm{C}$ twice with $2 X$ SSC, $0.1 \%$ (w/v) SDS, flow $10 \mathrm{~s}$ and hold $20 \mathrm{~s}$. Wash slides at $42{ }^{\circ} \mathrm{C}$ twice with $0.1 \mathrm{X} \mathrm{SSC}$, $0.1 \%$ SDS, flow $10 \mathrm{~s}$ and hold $20 \mathrm{~s}$. Wash slides at $42^{\circ} \mathrm{C}$ twice with $0.1 \mathrm{X}$ SSC, flow $10 \mathrm{~s}$ and hold $20 \mathrm{~s}$.

4. Dry slides by centrifugation (as in 4.6).

\section{Array quantification}

1. Wrap slides using a thin plastic film. Check slides for radioactive signal using a Geiger counter on its most sensitive setting. Expose slides onto a storage phosphor screen in an exposure cassette at room temperature for 10 to $90 \mathrm{~h}$ depending on signal strength.

NOTE: As sensitivity of Geiger counters vary from one instrument to another, exposure times must be optimized empirically.

2. Scan slide at $50 \mu \mathrm{m}$ resolution using a phosphorimager. Quantify and background-subtract radioactivity intensities at each probe spot using the free Image J software upgraded with a microarray profiler.

3. Organize and process data using an electronic spreadsheet. Generate heat maps using the conditional formatting tool. Represent for example each probe signal as a fraction (expressed in \%o) of the total probe signal.

\section{Representative Results}

Three independent biological replicates show that, under the tested growth conditions, all $M$. smegmatis tRNAs are expressed above background level (Figure 1). In this particular experiment, the observed standard deviation for each probe is comprised between $2 \%$ (Arg (TCT)) and $22 \%$ (Cys (GCA)) with a median at $5 \%$ (Figure 1). False changes between replicates are significantly influenced by factors such as array preparation and hybridization. High reproducibility is achieved through careful and consistent array printing as well as automated sample hybridization. There is a 5-fold difference between tRNA Ile (GAT) and tRNA (Val (TAC), the highest and lowest abundant species respectively. SPOt shows that the expression of tRNA isoacceptors is not uniform. Isoacceptors are species that hold the same amino acid but display different anticodon sequences and therefore decode different codons on mRNAs. For example, all Alanine isoacceptors are expressed at similar levels, whereas the highest and lowest Arginine accepting tRNAs differ by 3-fold. 


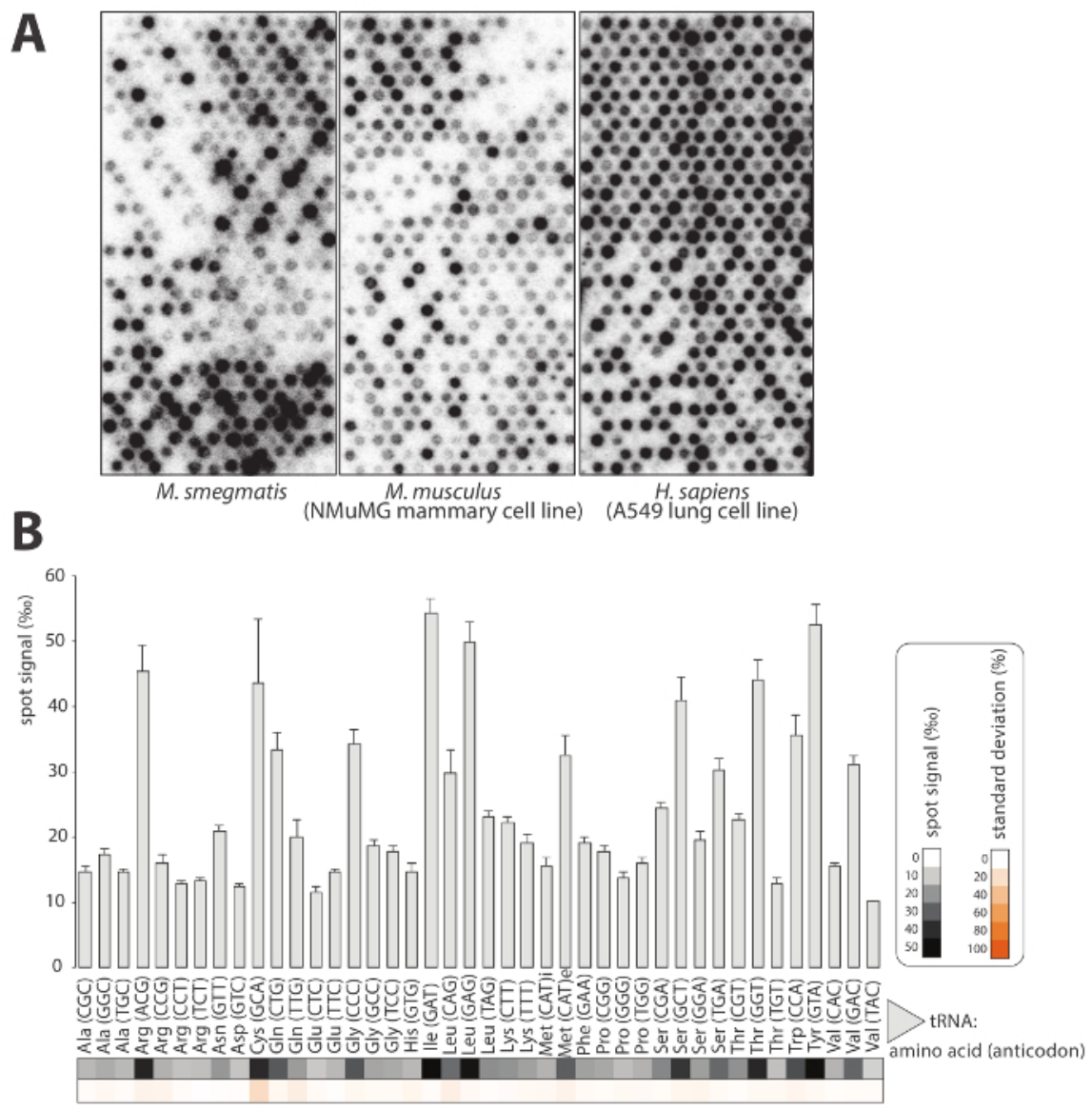

Figure 1: Representative tRNA macroarray results. (A) Scanned bacterial, mouse and Human macroarrays. M. smegmatis arrays use only 43 probes instead of 48 for mouse and Human. As a consequence, the bacterial array displays 40 empty spots (probes are replicated eight times on each array) that blend with the background. Most of them are clustered in the upper left corner. (B) Histogram and heatmap representations of tRNA profiles in M. smegmatis under optimal growth conditions. Probe signals are the average of three independent experiments (including bacterial growth, metabolic labeling, extraction and array hybridization) and are expressed as per thousand values of total tRNAs. Standard deviations for the three replicates are indicated as error bars and shades of orange on the histogram and heatmap respectively. Please click here to view a larger version of this figure.

\section{Discussion}

Beta particle emissions are known bacteriostatic and bactericidal agents ${ }^{14}$, however radiations in the tested range have no significant impact on cell fitness. Scintillation counting showed that the radioactivity is incorporated into $25 \%$ of total-cell extracts and subsequently, $1 \%$ of purified total RNAs.

The probes' identical sizes, comparable melting temperature and GC content, along with a uniform protocol for macroarray spotting, hybridization and quantification allows for unbiased measurement of tRNA levels directly from spot signals.

SPOt is reproducible and specific. Its large dynamic range and easily adjustable threshold allows profiling low abundant species, such as tRNAs associated with polysomes, simply by prolonging array exposure times ${ }^{9}$. After the initial investment for the necessary equipment, the running cost per sample, including consumables, averages $\$ 15$ per samples.

SPOt is applicable to any organism whose genome is available for probe design. Model organisms that are grown in vitro are ideal candidates for metabolic labeling. As mammalian genomes often encode many isodecoders (tRNAs that share identical anticodons but display small differences in their body sequences) probes need to be partially degenerated to preserve homogenous hybridization of close tRNA species. Finally, adherent mammalian cells yield typically lower amounts of total RNA compared to organisms grown in suspension such as M. smegmatis, so cultures need to be substantially scaled up.

\section{Disclosures}

No conflict of interest declared. 


\section{Acknowledgements}

This work was supported by a SC INBRE grant from the National Institute of General Medical Science - NIH (P20GM103499 to R.G.) and grants CA555536 \& CA154664 from the National Cancer Institute to P.H.H. This program was supported in part by a grant to the College of Charleston from the Howard Hughes Medical Institute through the Pre-college \& Undergraduate Science Education Program and by grants from the National Center for Research Resources (5 P20 RR016461) and the National Institute of General Medical Sciences (8 P20 GM103499) from the National Institutes of Health.

\section{References}

1. Emilsson, V. and Kurland, C.G. Growth rate dependence of transfer RNA abundance in Escherichia coli. EMBO J. 9(13):4359-66 (1990).

2. Dittmar, K.A., Goodenbour, J.M., Pan, T. Tissue-specific differences in human transfer RNA expression. PLoS Genet. 2 (12):e221 (2006).

3. Pavon-Eternod, M., Gomes, S., Geslain, R., Dai, Q., Rosner, M.R. and Pan, T. tRNA over-expression in breast cancer and functional consequences. Nucleic Acids Res. 37 (21):7268-80 (2009).

4. Giegé, R, Jühling, F, Pütz, J, Stadler, P, Sauter, C, Florentz, C. Structure of transfer RNAs: similarity and variability. Wiley Interdiscip Rev RNA. 3 (1):37-61 (2012).

5. Machnicka, M.A., Olchowik, A., Grosjean, H., Bujnicki, J.M. Distribution and frequencies of post-transcriptional modifications in tRNAs. RNA Biol. 11 (12):1619-29 (2014).

6. Torres, A.G., Piñeyro, D., Rodríguez-Escribà, M., Camacho, N., Reina, O., Saint-Léger, A., Filonava, L., Batlle, E., Ribas de Pouplana, L. Inosine modifications in human tRNAs are incorporated at the precursor tRNA level. Nucleic Acids Res. 43 (10):5145-57 (2015).

7. Cognat, V., Deragon, J.M., Vinogradova, E., Salinas, T., Remacle, C. and Maréchal-Drouard, L. On the evolution and expression of Chlamydomonas reinhardtii nucleus-encoded transfer RNA genes. Genetics. 179 (1):113-23 (2008).

8. Zheng, G., Qin, Y., Clark, W.C., Dai, Q., Yi, C., He, C. et al. Efficient and quantitative high-throughput tRNA sequencing. Nat Methods. 12 (9):835-7 (2015).

9. Grelet, S., McShane, A., Hok, E., Tomberlin, J., Howe, P.H. and Geslain, R. SPOt: A novel and streamlined microarray platform for observing cellular tRNA levels. PLoS One. 12 (5):e0177939 (2017).

10. Eriani, G., Karam, J., Jacinto, J., Morris Richard, E. and Geslain, R. MIST, a Novel Approach to Reveal Hidden Substrate Specificity in Aminoacyl-tRNA Synthetases. PLoS One. 10 (6):e0130042 (2015).

11. Grelet, S., McShane, A., Geslain, R., Howe, P.H. Pleiotropic Roles of Non-Coding RNAs in TGF- $\beta$-Mediated Epithelial-Mesenchymal Transition and Their Functions in Tumor Progression. Cancers. 9 (7) (2017).

12. Belanger, A.E., Hatfull, G.F. Exponential-phase glycogen recycling is essential for growth of Mycobacterium smegmatis. J Bacteriol. 181 (21):6670-8 (1999).

13. Chan, P.P., \& Lowe, T.M. GtRNAdb: A database of transfer RNA genes detected in genomic sequence. Nucl. Acids Res. 37 (Database issue):D93-D97 (2009).

14. Schmidt, C.F. The Effect of Radioactive Phosphorus upon a Suspension of Escherichia coli. J Bacteriol. 55 (5):705-10 (1948). 